\title{
Nonmodal energetics of resistive drift waves
}

\author{
Suzana J. Camargo \\ Universidade Estadual Paulista, Campus de Guaratinguetá, Avenida Dr. Ariberto Pereira da Cunha, 333, \\ 12500-000 Guaratinguetá, São Paulo, Brazil \\ Michael K. Tippett \\ Centro de Previsão do Tempo e Estudos Climáticos, Instituto Nacional de Pesquisas Espaciais, \\ Rodovia Presidente Dutra Km 40, 12630-000 Cachoeira Paulista, São Paulo, Brazil \\ Iberê L. Caldas \\ Instituto de Física, Universidade de São Paulo, Caixa Postal 66318, 05315-970 São Paulo, São Paulo, Brazil
}

(Received 12 February 1998)

\begin{abstract}
The modal and nonmodal linear properties of the Hasegawa-Wakatani system are examined. This linear model for plasma drift waves is nonnormal in the sense of not having a complete set of orthogonal eigenvectors. A consequence of nonnormality is that finite-time nonmodal growth rates can be larger than modal growth rates. In this system, the nonmodal time-dependent behavior depends strongly on the adiabatic parameter and the time scale of interest. For small values of the adiabatic parameter and short time scales, the nonmodal growth rates, wave number, and phase shifts (between the density and potential fluctuations) are time dependent and differ from those obtained by normal mode analysis. On a given time scale, when the adiabatic parameter is less than a critical value, the drift waves are dominated by nonmodal effects while for values of the adiabatic parameter greater than the critical value, the behavior is that given by normal mode analysis. The critical adiabatic parameter decreases with time and modal behavior eventually dominates. The nonmodal linear properties of the Hasegawa-Wakatani system may help to explain features of the full system previously attributed to nonlinearity. [S1063-651X(98)13308-1]
\end{abstract}

PACS number(s): 52.35.Kt, 52.25.Gj, 52.35.Ra

\section{INTRODUCTION}

A fundamental issue in fluid dynamics is the question of how laminar flows become unstable and eventually turbulent. A method of studying instabilities is to consider the time evolution of a perturbation of the laminar flow ([1], $\mathrm{p}$. 13). In general, the evolution of the perturbation is described by a nonlinear system. However, for small perturbations, the nonlinear system can be approximated by a linear system. When the background flow is time independent, an eigenmode ("normal mode") analysis of the linear system identifies exponentially growing and decaying perturbations. The dependence of these exponential growth rates on the geometry, the Reynolds number, and other parameters allows the theory to make predictions regarding the stability of laboratory flows.

Normal mode analysis has been applied to many fluid dynamic stability problems with great success. However, there are some notable cases where the results of normal mode analysis fail to correspond to observed temporal variation and spatial structure of real flows. In particular, normal mode analysis predicts a transition to turbulence for some flows at a much higher Reynolds numbers than that seen in experiment. Attributing this failure to the linearization of a nonlinear problem has led to the development of theories which modify or just eliminate the need for linearization such as the energy method ([1], p. 431).

Recent studies have shown that for many physically relevant problems, normal mode analysis only gives a partial description of the properties of the linear perturbation equa- tion [2,3]. When the eigenmodes of the linear system are not orthogonal and complete, or equivalently when the system is nonnormal, the general solution of the system may present a behavior quite different from that suggested by normal mode analysis. For example, perturbations in some nonnormal systems can be amplified by factors of thousands even when all the normal modes of the system are stable [4]. The possibility of amplification of perturbations in nonnormal flows has been known for a long time, but only recently have computational resources made it possible to calculate the magnitudes involved (see [2] and [5], and references therein). Recent applications of nonmodal analysis to physical problems include nonmodal growth in atmospheric flows [6], atmospheric turbulence models [7], the Orr-Sommerfeld equation [8], models for the transition to turbulence [9], and methods of controlling turbulence [10].

The study of instabilities in plasmas is heavily based on normal mode analysis ([11], p. 309) and variational methods ([12], p. 251). Nonlinear stability bounds have been obtained for magnetohydrodynamic flows (e.g., [13]), though the stability bounds are in general very low. The role of nonnormality in plasma stability has been studied in a few cases (e.g., $[14,15])$.

Drift-wave turbulence is considered to be a possible cause of anomalous transport in the cool plasma edge region of tokamaks $[16,17]$. In this work we use nonnormal analysis methods to study the linear properties of a drift-wave turbulence model. The Hasegawa-Wakatani model considered here $[18,19]$ has been extensively studied in bidimensional [20-22] and tridimensional numerical simulations [23,24]. 
The linearized Hasegawa-Wakatani equations have been examined using normal mode analysis (e.g., [20]). However, an analysis of the linear system including time-dependent, nonmodal behavior has not been made previously. The role of nonlinearity in drift-wave models is typically examined by comparing the linear behavior given by normal mode analysis with the nonlinear numerical simulation. Therefore a complete understanding of the linear behavior is fundamental to identifying truly nonlinear effects.

In Sec. II the nonlinear Hasegawa-Wakatani model is described. The linear bidimensional Hasegawa-Wakatani system is presented in Sec. III along with the quantities that will be used to measure its nonmodal behavior. The results are presented and discussed in Sec. IV. The finite-time behavior of solutions with optimally chosen initial conditions and with random initial conditions are calculated and compared with that of the normal modes. The importance of nonmodal growth mechanisms is demonstrated to depend strongly on the adiabatic parameter $\mathcal{C}$. The system presents strong nonmodal growth for small values of $\mathcal{C}$. For large values of $\mathcal{C}$, the nonmodal behavior of the system differs little from that given by normal mode analysis. For small values of $\mathcal{C}$ the nonnormal growth rates and phase shifts (between the density and potential fluctuations) are time dependent and differ greatly from previously obtained normal mode results. Likewise, the wave number at which maximum growth occurs is time dependent and differs from those predicted by the normal mode analysis. We hypothesize that some properties of the drift-wave turbulence previously attributed to nonlinear terms could also be strongly influenced by the nonnormal character of the linear system. Finally, a summary and conclusions are given in Sec. V. In the Appendix, details of the nonmodal analysis calculation are shown.

\section{NONLINEAR MODEL}

The model of our studies is the Hasegawa-Wakatani system $[18,19]$. We consider two-dimensional density and potential fluctuations, perpendicular to the static equilibrium magnetic field $\mathbf{B}=B \hat{\mathbf{z}}$; magnetic fluctuations are neglected. A nonuniform equilibrium density $n_{0}$ with density gradient $d n_{0} / d x$ in the negative $x$ direction is considered, such that the equilibrium density scale $L_{n}=n_{0} /\left|d n_{0} / d x\right|$ is constant. The ions are cold and the electrons are isothermal, $T_{i} \ll T_{e}$ $\equiv T$. Therefore temperature gradients and fluctuations are neglected, as well as finite Larmor effects. We assume that the fluctuation length scales satisfy the usual drift ordering $k_{\|}$ $\ll k_{\perp}$. The equations for the time evolution of the density and potential fluctuations are two coupled nonlinear equations given by $[18,19]$

$$
\begin{gathered}
\frac{\partial}{\partial t} \nabla_{\perp}^{2} \phi+\left(\hat{\mathbf{z}} \times \boldsymbol{\nabla}_{\perp} \phi\right) \cdot \nabla_{\perp} \nabla_{\perp}^{2} \phi=\mathcal{C}(\phi-n)+D^{\phi}, \\
\frac{\partial}{\partial t} n+\left(\hat{\mathbf{z}} \times \boldsymbol{\nabla}_{\perp} \phi\right) \cdot \nabla_{\perp} n+\frac{\partial \phi}{\partial y}=\mathcal{C}(\phi-n)+D^{n},
\end{gathered}
$$

where the usual dimensionless variables are

$$
x \rightarrow \frac{x}{\rho_{s}}, \quad y \rightarrow \frac{y}{\rho_{s}}, \quad t \rightarrow t \frac{c_{s}}{L_{n}}
$$

and the normalized potential and density fluctuations are

$$
\phi \rightarrow \frac{e \phi}{T} \frac{L_{n}}{\rho_{s}}, \quad n \rightarrow \frac{n}{n_{0}} \frac{L_{n}}{\rho_{s}},
$$

$\rho_{s}$ is the drift-wave dispersion scale $\left[\rho_{s}^{2}=c^{2} M_{i} T /\left(e^{2} B^{2}\right)\right]$; $c_{s}$ is the sound speed $\left(c_{s}^{2}=T / M_{i}\right)$.

The adiabaticity parameter $\mathcal{C}$, which couples the equations linearly and determines the character of the system, is defined as [20,21]

$$
\mathcal{C}=\frac{T}{n_{0} e^{2} \eta_{\|}} \frac{k_{\|}^{2}}{c_{s} / L_{n}},
$$

where $\eta_{\|}$is the resistivity of the plasma in the direction of the magnetic field. In the limit $\mathcal{C} \gg 1$, the electron response is almost adiabatic, meaning that the fluctuations of the density follow very nearly the fluctuations of the potential. In this limit, Eqs. (1) and (2) reduce to the Hasegawa-Mima equation [25]. In the opposite limit, $\mathcal{C} \ll 1$, Eq. (1) reduces to the Navier-Stokes equation, where density fluctuations are passively advected.

The viscous and diffusive dissipation terms $D^{\phi}$ and $D^{n}$, respectively, are chosen to have the form

$$
D^{\phi}=-\nu \nabla_{\perp}^{6} \phi, \quad D^{n}=-\nu \nabla_{\perp}^{4} n,
$$

in order to confine the dissipation to the smallest scales resolved in the system. The Hasegawa-Wakatani system is a simple model for drift-wave turbulence in a collisional plasma with a magnetic field without shear. It is an autonomous system describing the excitation and damping of modes in terms of a few collisional parameters, leading to a stationary level of turbulence without need of external forcing [20].

We usually choose time scales in our study of the order $t=10-100$, as we want to compare our work with previous numerical simulations of the bidimensional nonlinear Hasegawa-Wakatani system [20]. In those nonlinear numerical simulations, there are two phases, a linear phase followed by a nonlinear phase with a stationary turbulent regime. Since usually $k_{\|}$is not measured, $\mathcal{C}$ should be estimated from scaling laws conjectured for drift-wave dispersion. We consider a large interval of possible values for $\mathcal{C}$. In most cases, we choose $\mathcal{C}=10^{-5}, 10^{-3}, 0.1$, and 1 . When we consider the growth rates and their corresponding modes as functions of $\mathcal{C}, \mathcal{C}$ varies in the interval $10^{-5}-10$.

\section{LINEAR MODEL}

To study the linear properties of the system of Eqs. (1) and (2), we neglect the nonlinear terms and expand $\phi$ and $n$ in a double Fourier series in $x$ and $y$. For any wave number pair $\mathbf{k}=\left(k_{x}, k_{y}\right)$ the time evolution of the Fourier components of $\phi$ and $n$ has the form

$$
\frac{d}{d t} \mathbf{u}_{\mathbf{k}}=\mathbf{A}_{\mathbf{k}} \mathbf{u}_{\mathbf{k}}
$$




$$
\begin{gathered}
\mathbf{u}_{\mathbf{k}}=\left(\begin{array}{c}
\phi_{\mathbf{k}} \\
n_{\mathbf{k}}
\end{array}\right), \\
\mathbf{A}_{\mathbf{k}}=\left(\begin{array}{cc}
-\mathcal{C} / k^{2}-\nu_{\phi} k^{4} & \mathcal{C} / k^{2} \\
-i k_{y}+\mathcal{C} & -\mathcal{C}-\nu_{n} k^{4}
\end{array}\right), \\
k=\sqrt{k_{x}^{2}+k_{y}^{2}} .
\end{gathered}
$$

There is no coupling between distinct pairs of wave numbers. This property greatly reduces the numerical cost of analyzing the problem.

Assuming that the time dependence of the perturbations is exponential, e.g., $\sim e^{\gamma t}$, reduces Eq. (7) to the eigenvalue problem

$$
(\gamma \mathbf{l}-\mathbf{A}) \mathbf{u}=0,
$$

where $\mathbf{u}$ is the vector containing all the Fourier components $\mathbf{u}_{\mathbf{k}}$ and $\mathbf{A}$ is the block-diagonal matrix with entries $\mathbf{A}_{\mathbf{k}}$; if the number of modes in $k_{x}$ and in $k_{y}$ is $N$ then $\mathbf{u}$ is a vector of length $2 N^{2}$ and $\mathbf{A}$ is a $2 N^{2} \times 2 N^{2}$ matrix. Equation (11) has a nonzero solution if and only if $\gamma$ is an eigenvalue of $\mathbf{A}$. The normal mode growth rate $\beta_{0}=\operatorname{Re} \gamma$ is calculated by finding the eigenvalue of $\mathbf{A}$ with the largest real part.

Previous studies have given a normal mode analysis of the linear Hasegawa-Wakatani equations by solving Eq. (11) (e.g., [20]). An analysis of the approximate solutions of Eq. (11) or equivalently a nonnormal analysis of Eq. (7) has not been presented previously. The non-normal analysis allows time-dependent, nonmodal behavior rather than imposing an exponential time dependence.

The fundamental reason that Eq. (7) can present different behavior from that suggested by its normal mode analysis is that the matrix $\mathbf{A}$ is non-normal. That is to say, it does not have a complete set of orthogonal eigenvectors or equivalently it does not commute with its adjoint ([26], p. 313). Since both the notion of orthogonality and adjoint depend on the choice of inner product, it is necessary to use an inner product coming from a physically relevant norm. The most obvious choice of norm $\|\cdot\|$ is that coming from the total energy of the fluctuations $E$ given by

$$
E=\frac{1}{2} \int d^{2} x\left(\left|\nabla_{\perp}^{2} \phi\right|^{2}+n^{2}\right)=\frac{1}{2} \sum_{\mathbf{k}}\left(k^{2} \phi_{\mathbf{k}}^{2}+n_{\mathbf{k}}^{2}\right),
$$

an invariant of the purely nonlinear Hasegawa-Wakatani system [20]. It is a direct calculation to verify that the dynamical operator $\mathbf{A}$, which describes the linear evolution of the Hasegawa-Wakatani system is a nonnormal operator with respect to the energy norm. In this norm, $\left\|\mathbf{u}_{\mathbf{k}}\right\|$ corresponds to the energy density of the mode $\mathbf{k}$.

The range of behavior possible in this model and the need to account for both modal and nonmodal behavior can be seen by considering two extreme limiting values of $\mathcal{C}$. First, for $\mathcal{C} \gg 1$, neglecting all terms that do not contain $\mathcal{C}$ gives

$$
\frac{d}{d t}\left(\begin{array}{c}
k \phi_{\mathbf{k}} \\
n_{\mathbf{k}}
\end{array}\right)=\left(\begin{array}{cc}
-\mathcal{C} / k^{2} & -\mathcal{C} / k \\
\mathcal{C} / k & -\mathcal{C}
\end{array}\right)\left(\begin{array}{c}
k \phi_{\mathbf{k}} \\
n_{\mathbf{k}}
\end{array}\right) .
$$

This system is normal with respect to the energy inner product and its behavior is modal. The nondissipative limit, $\mathcal{C}$
$=0$, gives $\phi_{\mathbf{k}}=$ const and $n_{\mathbf{k}}=-i k_{y} t \phi_{\mathbf{k}}+$ const, nonmodal algebraic growth. For intermediate values of $\mathcal{C}$ we expect to see a mixture of modal and nonmodal behavior. Algebraic growth was also observed in nonlinear simulations of the tridimensional Hasegawa-Wakatani system [23,24].

The basic quantities used in our investigation of the linear Hasegawa-Wakatani system are the energy growth ratio $\xi(t)$ defined as the ratio of the fluctuation energy at time $t$ to the initial fluctuation energy:

$$
\xi(t)=\frac{\|\mathbf{u}(t)\|}{\|\mathbf{u}(0)\|},
$$

and its growth rate $\beta(t)$ defined by $\beta(t)=t^{-1} \ln \xi(t)$. The ratio $\xi(t)$ depends on the choice of initial fluctuations $\mathbf{u}(0)$ and time. We shall examine the energy growth ratio $\xi(t)$ for several choices of initial conditions using subscripts to distinguish these choices. We use the notation $\xi_{0}(t)$ to indicate that $\mathbf{u}(0)$ is taken to be the eigenvector of $\mathbf{A}$ whose real part is $\beta_{0}$. Then

$$
\xi_{0}(t)=e^{\beta_{0} t} .
$$

Note that $\beta_{0}=t^{-1} \ln \xi_{0}(t)$ is the normal mode growth rate and is constant in time. We use the notation $\xi_{1}(t)$ for the case where $\mathbf{u}(0)$ is chosen such that $\xi$ is maximized, i.e.,

$$
\xi_{1}(t)=\max _{\mathbf{u}(0)} \frac{\|\mathbf{u}(t)\|}{\|\mathbf{u}(0)\|} .
$$

The quantity $\xi_{1}(t)$ is related to $\mathbf{A}$ by $\xi_{1}(t)=\left\|e^{\mathbf{A} t}\right\|$ and is the largest factor by which the initial fluctuation energy can be amplified at time $t$. The exponential of a matrix can be defined, for instance, by a power series ([26], Sec. 11.3). If $\mathbf{A}$ is normal, $\xi_{1}(t)=\xi_{0}(t)$ at all times $t$ and the maximum growth is modal. More generally, $\xi_{1}(t) \geqslant \xi_{0}(t)$ and there are initial fluctuations that grow more than do normal modes. However, enhanced nonmodal growth cannot be maintained indefinitely and in the limit of large time the limit ([27], Problem 74)

$$
\lim _{t \rightarrow \infty} \beta_{1}(t)=\beta_{0}
$$

must be satisfied.

It is important to note that when the dominant behavior is genuinely nonmodal, the fluctuation initial value that produces maximum growth at a particular time will not necessarily be the fluctuation initial value that produces maximum growth at any other time. Therefore, to see the behavior of a single choice for the initial value of the fluctuations $\mathbf{u}(0)$, we define the following energy growth ratios: $\xi_{2}(t)$-the initial condition is chosen to produce maximum growth in the limit of large time, $\xi_{3}(t)$ - the initial condition is chosen to produce maximum growth at a specified finite time $\tau$, and $\xi_{4}(t)$ - the initial condition is chosen to produce maximum growth in the limit of small time. Recall again that for normal systems all these growth ratios coincide. The final measure of the energy growth $\xi_{5}(t)$ is obtained by considering the time evolution of suitably normalized random initial conditions. Note that by construction $\xi_{1}(t)$ is the upper bound 
(a)

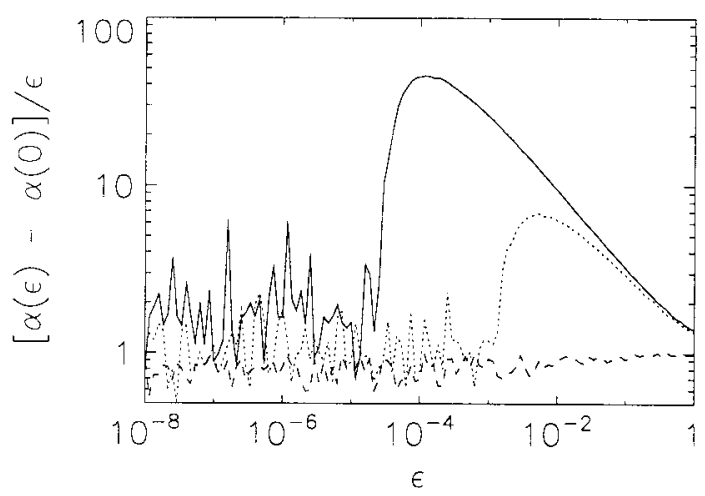

(c)

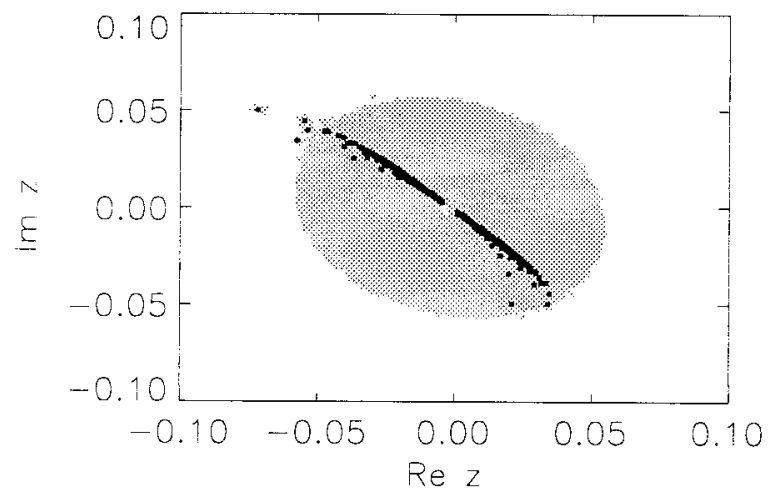

(b)

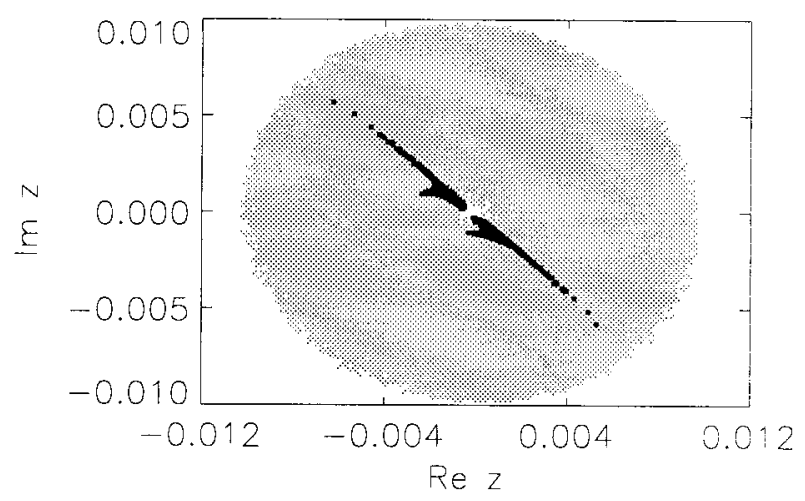

(d)

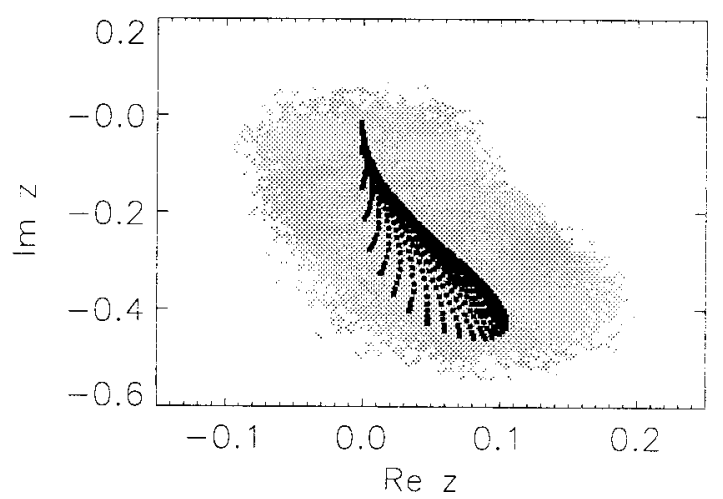

FIG. 1. (a) The quantity $[\alpha(\epsilon)-\alpha(0)] / \epsilon$ plotted as a function of $\epsilon$ for $\mathcal{C}=10^{-5}$ (solid line), $\mathcal{C}=10^{-3}$ (dotted line), and $\mathcal{C}=1$ (dashed line). The spectrum $\Lambda(\mathbf{A})$ (black) and $\epsilon$ pseudospectrum $\Lambda_{\epsilon}(\mathbf{A})$ (gray) plotted in the complex $z$ plane for (b) $\mathcal{C}=10^{-5}$ and $\epsilon=10^{-4},(\mathrm{c})$ $\mathcal{C}=10^{-3}$, and $\epsilon=3.2 \times 10^{-3}$, and (d) $\mathcal{C}=1$ and $\epsilon=10^{-1}$. (d) shows only the most unstable branch of the spectrum.

for all these quantities, $\xi_{1}(t) \geqslant \xi_{0,2,3,4,5}(t)$. Details of the calculation of these ratios are given in the Appendix.

In Sec. IV we investigate numerically the various energy growth ratios, emphasizing their dependence on $\mathcal{C}$ and on the time scale. The absence of mode coupling reduces the number of modes that must be considered in the numerical calculations. Our calculations show that for the time scales considered both the modal and nonmodal behavior of the system is determined by relatively low wave numbers. Once these dominant wave numbers are included in the model, adding more modes has no effect on the calculation of the quantities $\xi_{\{0-4\}}$. In most of our calculations, the number of modes is $N=32$. The quantity $\xi_{5}(t)$ depends on all the wave numbers in the system, as it is calculated assuming that the energy of the random initial conditions is homogeneous in Fourier space with unit total energy (see the Appendix). Taking $N$ $=32$ is therefore an assumption on the spectrum of the random initial condition. The value taken for the dissipation is, in most cases, $\nu=10^{-5}$ which is the order of magnitude considered previously in nonlinear numerical simulations [20].

\section{RESULTS}

\section{A. Spectra and pseudospectra}

In this section the transient growth properties of the dynamics are shown to be connected to the pseudospectrum of
A obtained by the approximate solution of the eigenvalue problem (11). The set of eigenvalues or spectrum of a matrix $\mathbf{A}$ is the set $\Lambda(\mathbf{A})$ of complex numbers $z$ such that $(z \mathbf{I}-\mathbf{A})$ is singular. In Figs. 1(b)-1(d), we plot in the complex $z$ plane the spectra (in black) of $\mathbf{A}$, for $\mathcal{C}=10^{-5}, \mathcal{C}=10^{-3}$, and $\mathcal{C}$ $=1$. The values of the modal growth rate $\beta_{0}=\max \operatorname{Re} \Lambda$ can be simply read from these figures. The modal growth rate has a strong dependence on $\mathcal{C}$, as already noted in [20]; the values of the spectra and the form of the spectra change with $\mathcal{C}$. The pseudospectrum of a non-normal matrix may provide more information than its spectrum $[8,28]$. The pseudospectrum is defined as follows. The complex number $z$ is in the $\epsilon$ pseudospectrum $\Lambda_{\epsilon}(\mathbf{A})$ if $z \in \Lambda(\mathbf{A}+\mathbf{E})$ for $\|\mathbf{E}\| \leqslant \epsilon$. Note that $\Lambda_{0}(\mathbf{A})=\Lambda(\mathbf{A})$. If $z \in \Lambda_{\epsilon}(\mathbf{A})$ then it is in some sense an approximate eigenvalue, in that there is some vector $\mathbf{u}$ such that $\|\mathbf{A u}-z \mathbf{u}\| \leqslant \epsilon$.

Analysis of the pseudospectrum gives a stronger condition on energy growth than does analysis of the spectrum. Just as the normal mode growth rate depends on how far the spectrum extends into the real half-plane, analogously, the nonmodal energy growth depends on how far the pseudospectrum of $\mathbf{A}$ extends into the right half plane. This idea is made explicit by defining the extension $\alpha(\epsilon)$ of the pseudospectrum into the right half plane by

$$
\alpha(\boldsymbol{\epsilon})=\max _{z \in \Lambda_{\boldsymbol{\epsilon}}(\mathbf{A})} \operatorname{Re} z .
$$


Note that $\alpha(0)=\beta_{0}$ is the growth rate given by normal mode analysis. The first result using $\alpha(\epsilon)$ is that $\xi_{1}(t)=e^{\beta_{0} t}$ if and only if for all $\epsilon>0, \alpha(\epsilon)-\alpha(0) \leqslant \epsilon$, that is to say, if a perturbation of size $\boldsymbol{\epsilon}$ to $\mathbf{A}$ moves its eigenvalues by at most a distance $\epsilon$ further into the real half plane [8]. Secondly, it can be shown that [8]

$$
\max _{t \geqslant 0} \xi_{1}(t)=\max _{t \geqslant 0}\left\|e^{\mathbf{A} t}\right\| \geqslant \max _{\epsilon>0} \frac{\alpha(\epsilon)-\alpha(0)}{\epsilon} e^{\beta_{0} t} .
$$

Hence, if $[\alpha(\epsilon)-\alpha(0)] / \epsilon>1$ there are fluctuations that grow more than predicted by normal mode analysis.

The calculation of the $\epsilon$ pseudospectra is computationally expensive. An estimate of $\Lambda_{\epsilon}(\mathbf{A})$ can be obtained relatively inexpensively using the following Monte Carlo approach. The eigenvalues of $(\mathbf{A}+\mathbf{E})$ are calculated where $\|\mathbf{E}\|=\boldsymbol{\epsilon}$ and $\mathbf{E}$ is a complex matrix whose entries are independently distributed Gaussian random variables with mean zero and unit variance. Repeating this calculation for many realizations of E gives an estimate of $\Lambda_{\epsilon}(\mathbf{A})$. Figures 1(b)-1(d) show Monte Carlo estimates of the pseudospectrum (in gray) using ten realizations for particular values of $\epsilon$. In Fig. 1(a) the quantity $[\alpha(\epsilon)-\alpha(0)] / \epsilon$ estimated by the Monte Carlo method using 50 realizations is plotted. Figure 1(a) shows that the cases $\mathcal{C}=10^{-5}$ and $\mathcal{C}=10^{-3}$ produce transient growth that is at least a factor of, respectively, 45 and 6.8 greater than the normal mode exponential growth [see Eq. (19)]. For $\mathcal{C}=1,[\alpha(\epsilon)-\alpha(0)] / \epsilon \approx 1$, the same as for a normal matrix. This result combined with the previous remarks shows that for the case $\mathcal{C}=1$, the pseudospectrum does not predict energy growth greater than that given by normal mode analysis.

For the cases $\mathcal{C}=10^{-5}$ and $\mathcal{C}=10^{-3}$, the quantity $[\alpha(\epsilon)$ $-\alpha(0)] / \epsilon$ is found to be a function of $\epsilon,[\alpha(\epsilon)-\alpha(0)] / \epsilon$ $\approx O\left(\epsilon^{-p}\right)$, for values of $\epsilon$ that are not too small $\left(10^{-4} \leqslant \epsilon\right.$ $\leqslant 1$ ) with $p=0.35$ and $p=0.40$, respectively. Such nonanalytic (fractional power) dependence of the eigenvalues on perturbation size is typical of non-normal matrices ( [29], p. 77). For very small values of $\epsilon$, the behavior is more complex. A reason for there being two types of behavior is that the most sensitive eigenvalues (i.e., ones that make $[\alpha(\epsilon)-\alpha(0)] / \epsilon$ large) are not the most unstable eigenvalues [i.e., ones close to $\alpha(0)=\beta_{0}$ ]. For relatively large values of $\boldsymbol{\epsilon}$, perturbations to the most sensitive eigenvalues of $\mathbf{A}$ dominate the calculation of $\alpha(\epsilon)$. However, if the most sensitive eigenvalues are not the most unstable ones, then for small enough values of $\epsilon,[\alpha(\epsilon)-\alpha(0)] / \epsilon$ will reflect only the properties of the most unstable eigenvalues. Numerical calculations (not shown here) support this explanation. The relevant consequence of this point is that enhanced nonmodal growth is due to a different part of the spectrum (different wave numbers) than that which produces maximum modal growth.

\section{B. Energy growth ratios}

We now examine in detail the various energy growth ratios. Figure 2 shows the time evolution of the energy growth ratios $\xi_{\{0-5\}}(t)$ for $\mathcal{C}=10^{-5}, 10^{-3}, 0.1$, and 1.0. First, we discuss some general features of the modal growth $\xi_{0}(t)$ and maximum growth $\xi_{1}(t)$ curves. For large time the relation $\beta_{1}(t)=\beta_{0}$ must hold, i.e., the curves $\xi_{0}(t)$ and $\xi_{1}(t)$ must be parallel. The time required for $\beta_{1}(t)$ to approximate $\beta_{0}$ depends on the adiabaticity parameter $\mathcal{C}$; for large $\mathcal{C}(0.1$ and $1.0), \xi_{0}(t)$ and $\xi_{1}(t)$ are virtually indistinguishable for all values of $t$. Figures 2(a) and 2(b) show that for $t<50$ and small $\mathcal{C}, \beta_{1}(t)$ and $\beta_{0}(t)$ are different. For the case of $\mathcal{C}$ $=10^{-5}, \xi_{0}(t)$ and $\xi_{1}(t)$ are still not parallel at $t=100$; for $\mathcal{C}=10^{-3}$, the energy ratio growth rate relaxes to the modal one at about $t=50$. Increased nonmodal small-time growth causes the difference between the curves $\xi_{1}(t)$ and $\xi_{0}(t)$ at $t=100$ to be $O(100)$ and $O(10)$ for $\mathcal{C}=10^{-5}$ and $\mathcal{C}=0.1$, respectively. We note that the lower bounds obtained from the pseudospectra and Eq. (19) are satisfied.

The energy growth ratio curve $\xi_{2}(t)$ shows the time evolution of the energy of fluctuations whose initial values are chosen so that $\xi$ coincides with $\xi_{1}(t)$ for large time. As shown in the Appendix, the wave number $\mathbf{k}$ of these fluctuations is the same as that of the dominant modal instability. However, the partitioning of $\phi_{\mathbf{k}}$ and $n_{\mathbf{k}}$ is such that enhanced (compared to modal) growth is achieved [30]. In general, the limit $\xi_{2}(t) \rightarrow \xi_{1}(t)$ is satisfied only in the limit of large time. However, for this system, only for the case $\mathcal{C}=10^{-3}$ is there visible difference between the $\xi_{2}(t)$ and $\xi_{1}(t)$ curves. An explanation for this behavior is that for $\mathcal{C}=10^{-3}$ the wave number of the modal instability presents relatively weak nonmodal instability while in the other cases, the wave number of the modal instability also presents a strong nonmodal instability. In the next section we examine in more detail the wave number dependence and confirm this explanation [see Fig. 3(b)]. The energy growth ratio $\xi_{3}(t)$ shows the time evolution of fluctuations whose initial values are chosen so that $\xi_{3}(\tau)=\xi_{1}(\tau)$ for $\tau=100 / 3$. For the case $\mathcal{C}=10^{-3}$ and for $t>50, \xi_{3}(t)$ grows with a rate less than the maximum modal growth rate, indicating in this case that the wave number leading to maximal finite-time growth is different from the wave number at which the maximal modal growth occurs. Only for the case $\mathcal{C}=10^{-3}$ is there visible difference between the $\xi_{1}(t)$ and $\xi_{3}(t)$ curves for the same reasons mentioned above.

The curve $\xi_{4}(t)$ shows the evolution of fluctuations with maximum initial growth, i.e., the curve $\xi_{4}(t)$ is tangent to $\xi_{1}(t)$ at $t=0$. Maximum initial growth does not lead to longtime maximum growth. In all the cases, $\xi_{4}(t)$ eventually grows at a rate less than the modal growth rate $\beta_{0}$. This behavior implies that for all the values of $\mathcal{C}$ considered, the normal modal analysis does not identify the wave numbers which present maximum short-time nonmodal growth. For the case $\mathcal{C}=10^{-5}$ the growth of $\xi_{4}(t)$ is not too much less than $\xi_{1}(t)$. Later we show that this is due to the relatively weak dependence of the nonmodal growth rate on the wave number.

A curve of special interest is that of $\xi_{5}(t)$, which corresponds to the time evolution of the energy averaged over homogeneous, uncorrelated random initial conditions. Intuitively, this scenario would seem to be a reasonable model of what happens in experiments; all modes are excited, rather than one particular mode. Looking at Fig. 2, we note that for small values of $\mathcal{C}$ and small time, $\xi_{5}$ follows well the $\xi_{1}$ curve. This behavior suggests that for these values of $\mathcal{C}$ and time scales, nonmodal growth is not isolated to a few wave 
(a)

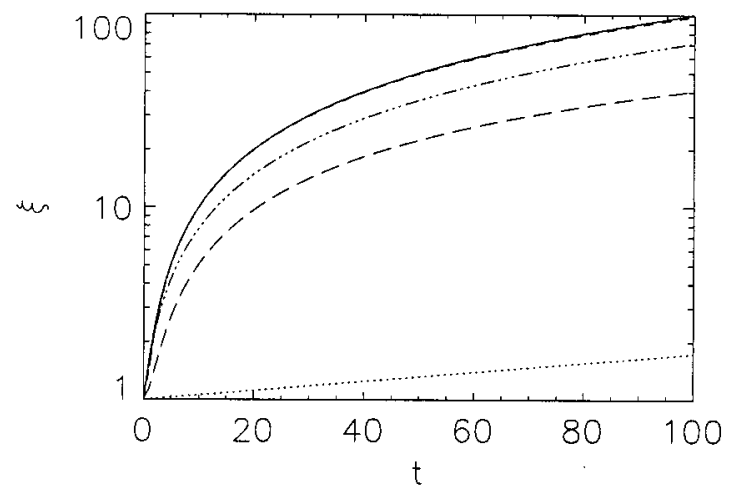

(c)

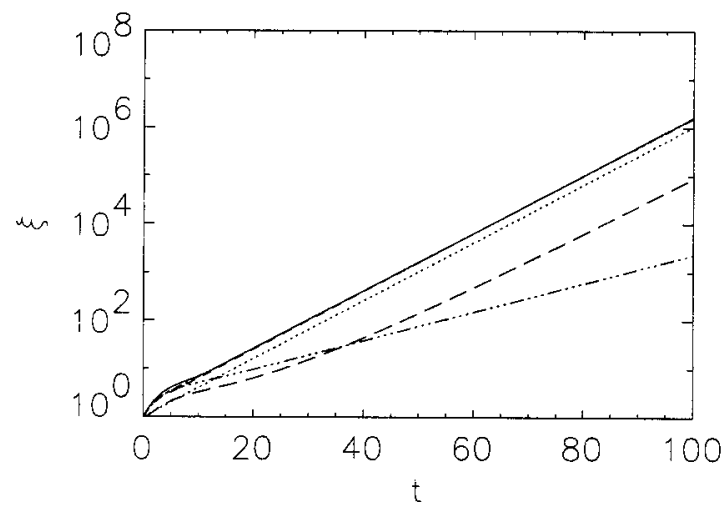

(b)

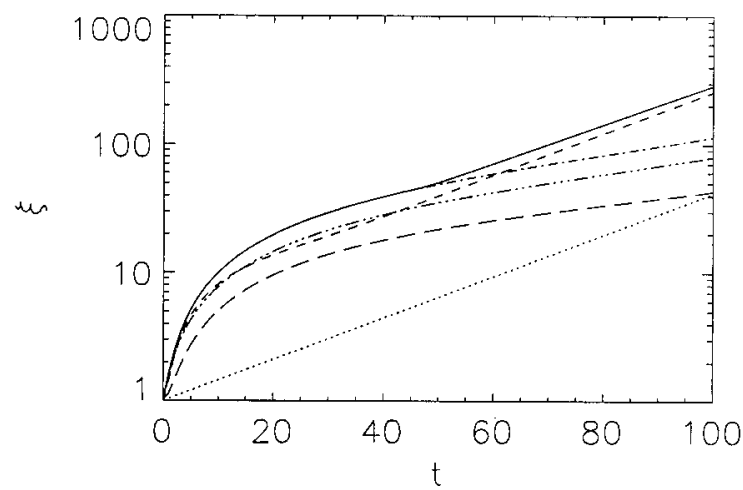

(d)

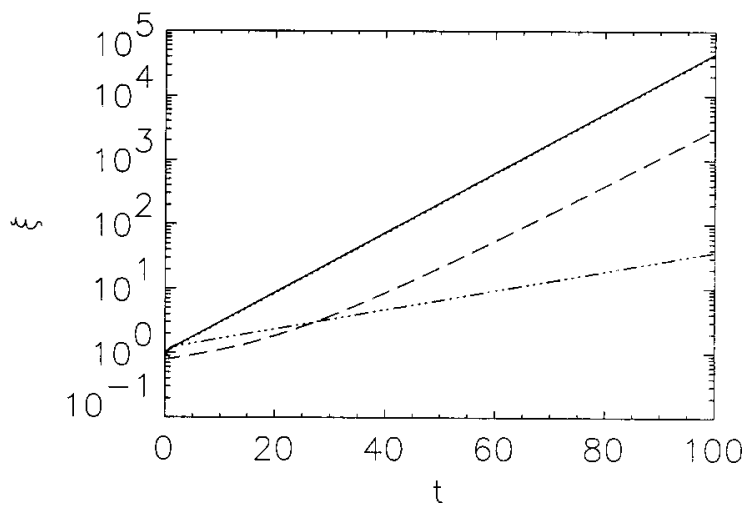

FIG. 2. Time evolution of the norms $\xi_{\{0-5\}}(t)$ (dotted, solid, dash, dash-dot, dash-dot-dot-dot, and long dash) for (a) $\mathcal{C}=10^{-5},(\mathrm{~b}) \mathcal{C}$ $=10^{-3}$, (c) $\mathcal{C}=0.1$, and (d) $\mathcal{C}=1$.

numbers but is a broad-spectrum phenomena, as will be confirmed later. Eventually, $\xi_{5}(t)$ must be parallel to $\xi_{0}$ as modal behavior dominates. In general, $\xi_{5}$ will be less than $\xi_{0}$ since the average is over all wave numbers and modal instability is found only at a few wave numbers. Figure 2(b) shows quite clearly the transition of $\xi_{5}$ from nonmodally dominant to modally dominant behavior [not shown here but eventually $\xi_{5}(t)$ is indeed parallel to $\left.\xi_{0}(t)\right]$. Figures 2(c) and 2(d) for $\mathcal{C}=0.1$ and $\mathcal{C}=1$, respectively, show that without broad-spectrum initial nonmodal growth, the expected energy of the random initial condition requires some time before the effects of the modal instability begin to be seen. This phenomenon of uncorrelated random initial conditions requiring time to "organize" before growing is well known in the meteorological literature and has generated several techniques for calculating "optimal" random initial conditions for use in ensemble forecasting [31,32]. Again in this case as the modal instabilities are confined to a few wave numbers and the average is over all wave numbers, $\xi_{5}$ is less than $\xi_{1}$.

In summary, the general features are that for small values of $\mathcal{C}$ the normal mode growth rate and associated time scale does not give a complete picture of the linear system; nonmodal growth is larger than modal growth. Second, in all cases the normal mode analysis does not identify the wave number at which maximum initial growth occurs. Third, examining the response to random initial conditions shows that the nonmodal behavior is robust for the small $\mathcal{C}$ case and that for large $\mathcal{C}$ the presence of damped modes lengthens the time it takes for the initially unorganized system to produce modal growth.

An important point is that for $\mathcal{C}=10^{-5}$, the modal growth rate is small, suggesting a long time scale, but the nonmodal growth is in reality much faster, as can be seen by the length of the nonmodal phase [compare $\xi_{0}$ and $\xi_{1}$ in Fig. 2(a)]. Similar dependence on $\mathcal{C}$ was observed when we studied the nonlinear turbulent system, for small $\mathcal{C}$ a saturated turbulent state was reached rather fast, while it took a long time to reach a saturated state for large values of $\mathcal{C}$ [20]. Therefore the non-normality might explain some features of the linear phase of a turbulent system.

\section{Growth rates}

A number of the features seen in the energy growth ratio curves of the preceding section can only be explained by a more detailed examination of the wave number dependence of the growth rates $\beta$. In Fig. 3 we show $\beta_{1}(t) \quad(t=10, t$ $=50$, and $t=100)$ and $\beta_{0}$ as functions of $k_{y}$ for $k_{x} \equiv 0$ and $\mathcal{C}=10^{-5}, \mathcal{C}=10^{-3}, \mathcal{C}=0.1$, and $\mathcal{C}=1$. The magnitude of the finite-time growth rates for small $\mathcal{C}$ and $t=10$ are slightly larger than those for $\mathcal{C}=0.1$ (approximately the $\mathcal{C}$ that gives the maximum modal growth rate). This dependence on $\mathcal{C}$ is exactly the opposite of that seen in the modal growth rate which peaks for $\mathcal{C} \sim 0.1$ and decreases on both 
(a)

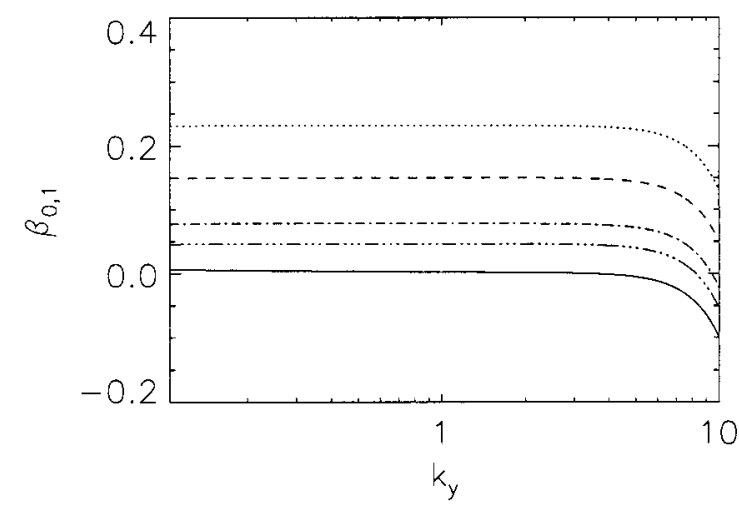

(c)

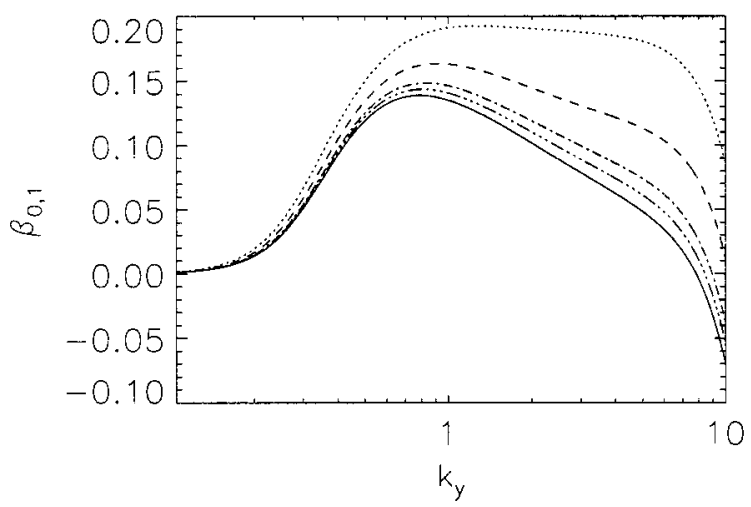

(b)

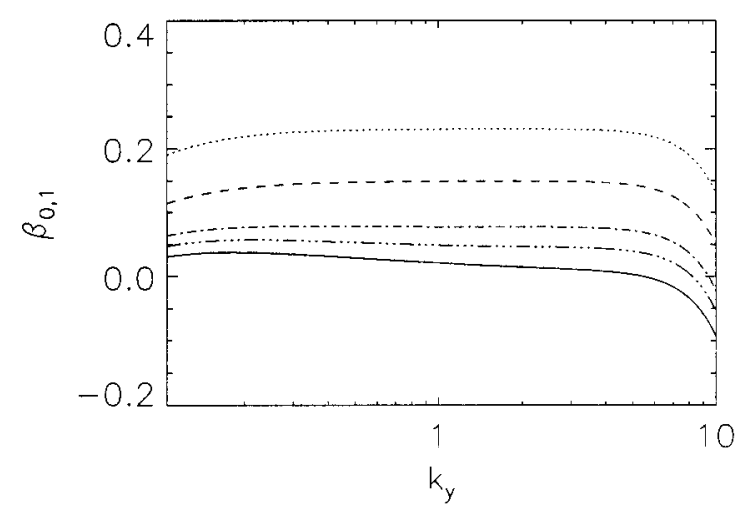

(d)

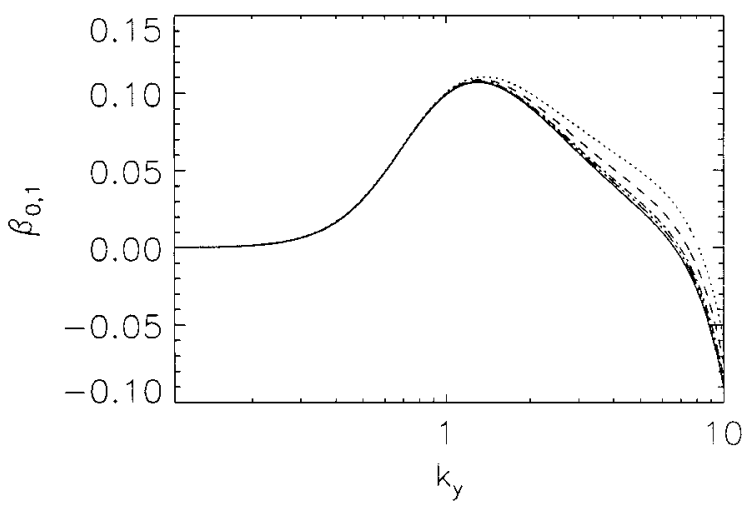

FIG. 3. The growth rates $\beta_{1}(t)[t=10$ (dotted); $t=20$ (dashed); $t=50$ (dashed-dot); $t=100$ (dash-dot-dot-dot) $]$ and $\beta_{0}$ (solid) as functions of $k_{y}$ for (a) $\mathcal{C}=10^{-5}$, (b) $\mathcal{C}=10^{-3}$, (c) $\mathcal{C}=0.1$, and (d) $\mathcal{C}=1$.

sides. Also as was suggested by the behavior of $\xi_{5}(t)$, the nonmodal growth for small $\mathcal{C}$ is a broad-spectrum phenomenon. As the time $t$ increases, the limit $\beta_{1}(t) \rightarrow \beta_{0}$ must hold. The rate of convergence of $\beta_{1}(\tau)$ to $\beta_{0}$ depends on $\mathcal{C}$. For small $\mathcal{C}$ the convergence is slow; for $\mathcal{C}=10^{-5}$ and $\mathcal{C}$ $=10^{-3}$ the $\beta(t=100)$ and $\beta_{0}$ curves are quite distinct. As $\mathcal{C}$ increases, the time scale for convergence becomes smaller. Also as $\mathcal{C}$ increases this convergence is nonuniform in $k_{y}$; $\beta_{1}(t) \rightarrow \beta_{0}$ first for small values of $k_{y}$.

The fact that the modes corresponding to large $k_{y}$ are in reality growing (i.e., are not damped) for small values of $\mathcal{C}$

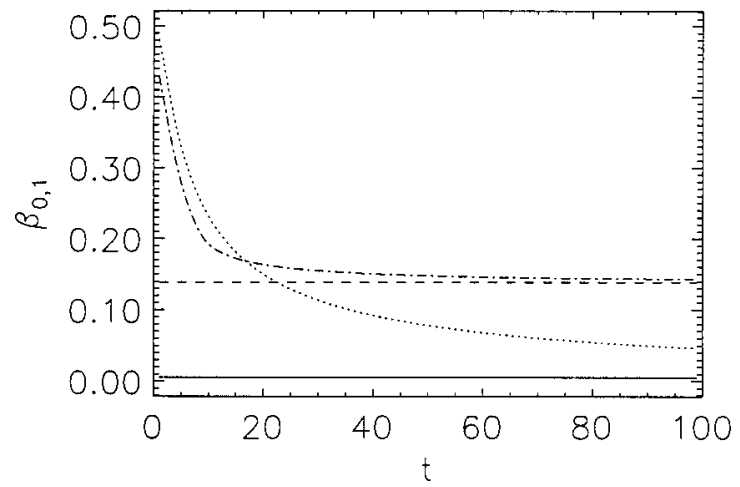

FIG. 4. The growth rates $\beta_{1}(t)$ and $\beta_{0}$, for $\mathcal{C}=10^{-5}$ (dotted; solid) and $\mathcal{C}=0.1$ (dot-dash; dashed). even as larger times are considered [see Fig. 3(a)] may be significant in nonlinear numerical simulations of drift-wave turbulence where there is coupling between modes. If these modes were damped, a certain spatial resolution and dissipation would be sufficient to study that case. However, if these modes are in reality growing, the spatial resolution and dissipation will not be adequate and could adversely influence the numerical simulations.

In Fig. 4 we compare $\beta_{0}$ and $\beta_{1}$ as functions of time for two values of $\mathcal{C}, \mathcal{C}=10^{-5}$ and $\mathcal{C}=0.1$. The influence of the parameter $\mathcal{C}$ on the modal growth rate $\beta_{0}$ is very strong. However, for small time $(t<20)$ the nonmodal growth rates for the two cases are comparable; in both cases there is enhanced nonmodal growth. To compare the effect of the hyperviscous dissipation $\nu$ on the growth rates $\beta_{0}$ and $\beta_{1}$, we repeat this calculation in Fig. 5 . We notice that for $\mathcal{C}$ $=10^{-5}[$ Fig. $5(\mathrm{a})]$ the difference of the growth rates $\beta_{0}$ and $\beta_{1}$ for $\nu=10^{-5}$ and $\nu=0.1$ is very small. By comparison, for $\mathcal{C}=0.1$ the difference of the growth rates for these two different values of $\nu$ is bigger, especially for smaller times. Therefore we can conclude that the influence of dissipation is more important in the adiabatic regime (bigger values of $\mathcal{C}$ ) and for smaller times. This role of viscosity is to be expected since for small $\mathcal{C}$ both the modally and nonmodally unstable wavelengths are long and while for large $\mathcal{C}$ they are small (see Fig. 3). 
(a)

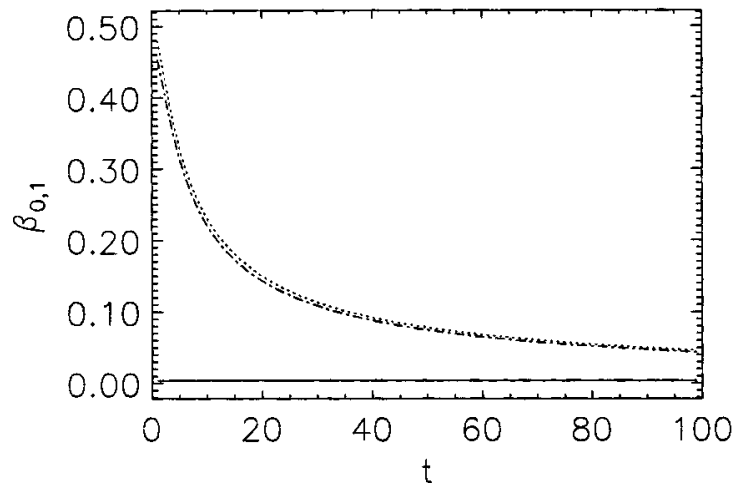

(b)

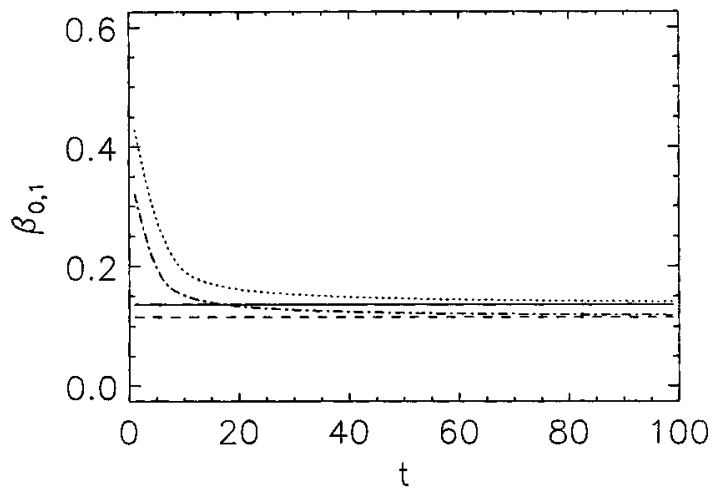

FIG. 5. The growth rates $\beta_{1}(t)$ and $\beta_{0}$ plotted as functions of time for $\nu=10^{-5}$ (dotted; solid) and $\nu=10^{-1}$ (dot-dashed; dashed) with (a) $\mathcal{C}=10^{-5}$ and (b) $\mathcal{C}=0.1$.

In Fig. 6(a) we show the growth rates $\beta_{0}$ and $\beta_{1}(t) \quad(t$ $=10, t=20, t=50$, and $t=100)$ as functions of $\mathcal{C}$. For large values of $\mathcal{C}(\mathcal{C}>1) \quad \beta_{0}$ and $\beta_{1}$ coincide for all values of $t$. For small values of $\mathcal{C}, \beta_{0}$ differs greatly from $\beta_{1}(t)$. For small enough $\mathcal{C}, \beta_{1}(t)$ does not depend on $\mathcal{C}$ and the $\mathcal{C}=0$ result of pure algebraic growth $\sim k_{y} t$ is recovered, as already observed in [23,24]. Figure 6(b) shows the wave numbers corresponding to the growth rates in Fig. 6(a) as a function of $\mathcal{C}$. We denote by $k_{0}^{\max }$ and $k_{1}^{\max }(t)$ the perpendicular wave number corresponding to the growth rates $\beta_{0}$ and $\beta_{1}(t) \quad(t$ $=10, t=20, t=50$, and $t=100)$. The behavior we see is the following. At a particular time $t$, the maximum growth wave number is the same as the maximum modal growth wave number, $k_{1}^{\max }(t) \approx k_{0}^{\max }$ for values of the adiabatic parameter $\mathcal{C}$ above some cutoff $\mathcal{C}_{\text {crit }}(t)$. However, if the adiabatic parameter is less than the critical one, $\mathcal{C}<\mathcal{C}_{\text {crit }}(t)$, then the maximum growth wave number is the same as the short-time maximum energy growth wave number, $k_{1}^{\max }(t) \approx k_{1}^{\max }(10)$. For values of $\mathcal{C}$ near the cutoff there is a transition region connecting the modal and nonmodal behavior. The cutoff value $\mathcal{C}_{\text {crit }}(t)$ is a decreasing function of time.

(a)

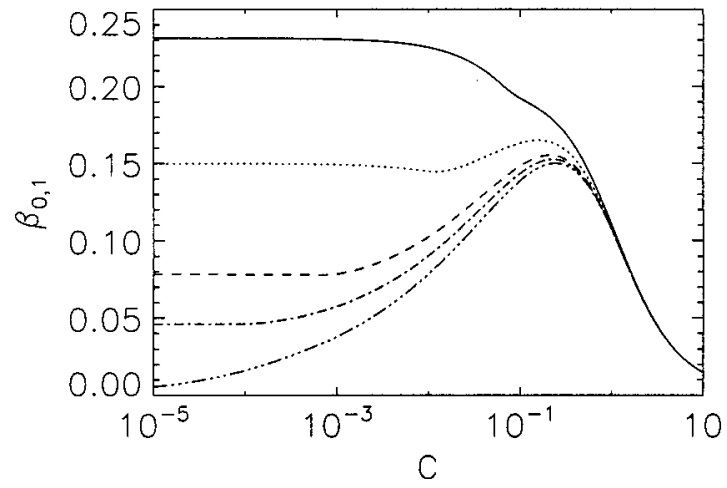

\section{Phase shifts}

A quantity of interest for transport studies is the the phase shift $\delta_{\mathbf{k}}$ between $n_{\mathbf{k}}$ and $\phi_{\mathbf{k}}$ defined by $[20,33]$

$$
\delta_{\mathbf{k}}=\operatorname{Im} \ln n_{\mathbf{k}}^{\dagger} \phi_{\mathbf{k}} \text {. }
$$

In nonlinear numerical simulations, the phase shift has been calculated by averaging Eq. (20) over realizations or in time. A normal mode linear phase shift $\delta_{\mathbf{k}}^{0}$ is calculated by taking $n_{\mathbf{k}}$ and $\phi_{\mathbf{k}}$ to be the components of the modal instability with wave number k. In analogy, a nonmodal finite-time phase shift $\delta_{\mathbf{k}}^{1}(t)$ can be calculated by using the components of the dominant nonmodal instability with wave number k. In Fig. 7 we compare the linear phase shifts $\delta_{\mathbf{k}}^{0}$ and $\delta_{\mathbf{k}}^{1}(t)$ for $k_{x}$ $\equiv 0$ for various values of $\mathcal{C}$ and $t$. For all values of $\mathcal{C}$ considered [Figs. $7(a)-7(d)]$ the nonmodal phase shift is initially larger than the modal one and for larger times they tend to have the same values. The larger the value of $\mathcal{C}$ the faster this agreement is reached; for $\mathcal{C}=1$ [Fig. $7(\mathrm{~d})]$ both phase shifts already coincide for all values of $k_{y}$ at $t=10$. In contrast, for $\mathcal{C}=10^{-5}$ [Fig. 7(a)] the phase shifts still differ at a time $t$ $=100$. The nonmodal phase shift first agrees with the

\section{(b)}

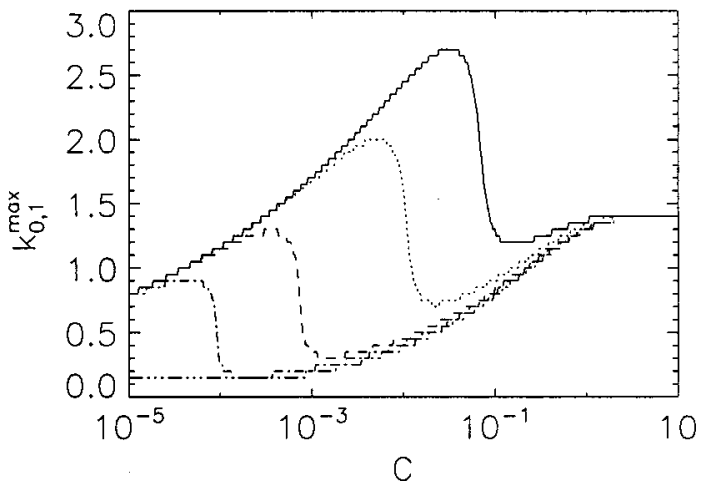

FIG. 6. (a) The growth rates $\beta_{0}$ (dot-dot-dot-dash) and $\beta_{1}(t)$ for $t=10$ (solid), $t=20$ (dotted), $t=50$ (dashed), and $t=100$ (dot-dash) plotted as functions of $\mathcal{C}$. (b) Values of $k_{0}^{\max }$ and $k_{1}^{\max }(t)$ for the same values of $t$ (and line styles) plotted as a function of $\mathcal{C}$. 
(a)

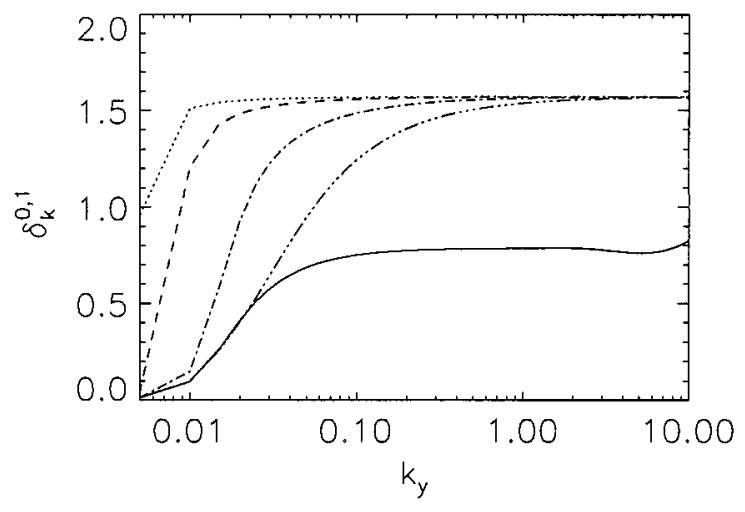

(c)

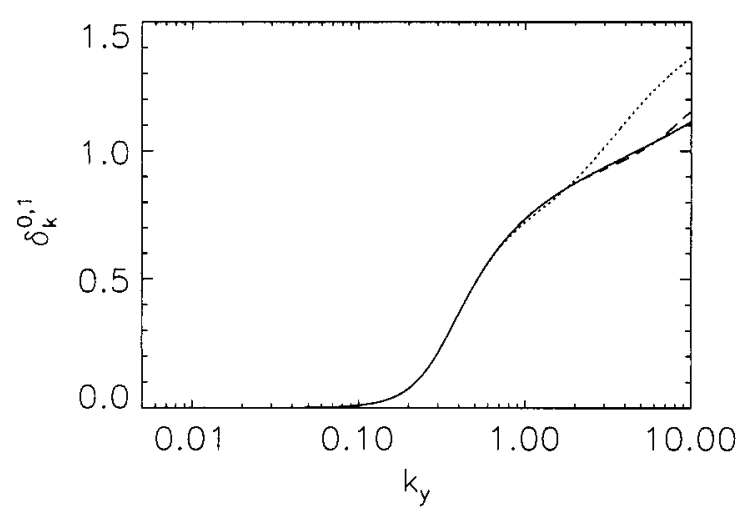

(b)

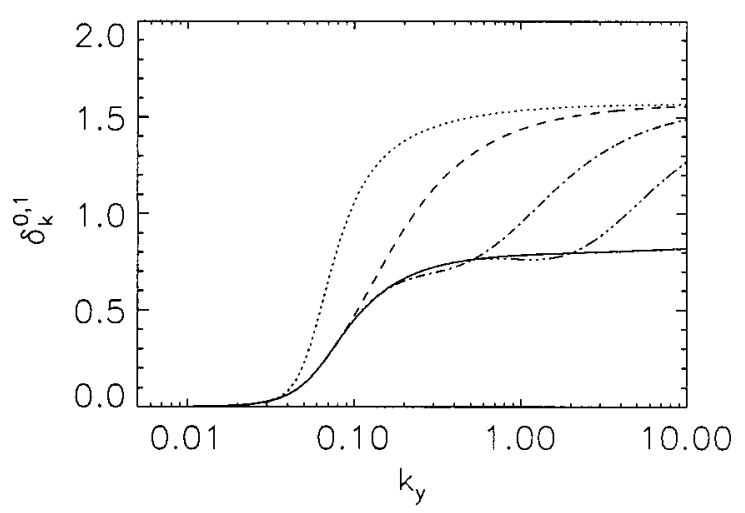

(d)

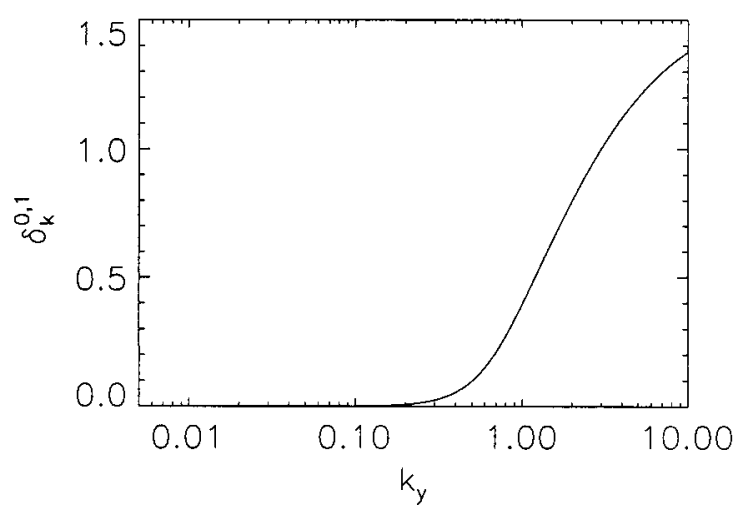

FIG. 7. Normal mode linear phase shift $\delta_{\mathbf{k}}^{0}$ and nonmodal linear phase shift $\delta_{\mathbf{k}}^{1}(t) \quad(t=10, t=20, t=50$, and $t=100)$ as a function of $k_{y}$ for (a) $\mathcal{C}=10^{-5}$, (b) $\mathcal{C}=10^{-3}$, (c) $\mathcal{C}=0.1$, and (d) $\mathcal{C}=1$. Here $\rho_{s}=0.005, N=2000$, and $k_{x} \equiv 0$.

modal phase shift for small values of $k_{y}$ and this agreement reaches larger values of $k_{y}$ for longer times.

The linear phase shift $\delta_{\mathbf{k}}^{5}(t)$ found by calculating the expected value of Im $\ln n_{\mathbf{k}}^{\dagger} \phi_{\mathbf{k}}$ is shown in Fig. $8 ; \delta_{\mathbf{k}}^{5}(t)$ is calculated in the same fashion as $\xi_{5}(t)$. In the limit of large time $\delta_{\mathbf{k}}^{5}(t)$ also goes to the normal mode linear phase shift as does $\delta_{\mathbf{k}}^{1}(t)$ (see Fig. 7). The finite-time values $\delta_{\mathbf{k}}^{5}(t)$ are quite different from those calculated in Fig. 7 for small values of $\mathcal{C}$ and small wave numbers $k_{y} . \delta_{\mathbf{k}}^{1}(t)$ and $\delta_{\mathbf{k}}^{5}(t)$ only differ for $\mathcal{C}=10^{-5}$ and $\mathcal{C}=10^{-3}$ and for wave numbers $k_{y}<0.1$. In these cases $\delta_{\mathbf{k}}^{5}(t)$ is smaller than the normal mode linear phase shift, while $\delta_{\mathbf{k}}^{1}(t)$ is larger than the latter. For $\mathcal{C}=0.1$ time dependence is seen only for short times and large wave numbers. For $\mathcal{C}=1$ the phase shift is time independent for the time scales shown.

The phase shifts obtained here can be compared to the nonlinear phase shifts calculated in nonlinear numerical simulations for $\mathcal{C}=0.1$ and $\mathcal{C}=1$ [20]. The linear and nonlinear phase shifts are very different for $\mathcal{C}=0.1$; in this case, the nonlinear phase shift is strongest at low $k$ and drops towards zero elsewhere, a tendency that is thought to be due to the turbulent advection, which tends to randomize the relationship between $n$ and $\phi[20]$. For $\mathcal{C}=1$ the nonlinear and linear phase shifts have the same dependence on $k$, though the nonlinear phase shift is still lower than the linear ones. For initial random conditions (with a chosen covariance) the ensemble averaged phase shifts $\delta_{5}^{\mathbf{k}}$ are initially smaller than the normal mode linear phase shift $\delta_{0}^{\mathbf{k}}$ for small values of $k_{y}$, which confirms this tendency that randomization diminishes the phase shifts.

\section{CONCLUSIONS}

We have examined the modal and nonmodal behavior of the linear Hasegawa-Wakatani drift-wave model. Nonmodal behavior plays a role in this system because it is non-normal, i.e., it does not have a complete set of orthogonal eigenvectors. The key parameter in the system is the adiabatic parameter $\mathcal{C}$. For $\mathcal{C} \gg 1$, the eigenvectors are complete and orthogonal with respect to the energy inner product; normal mode analysis gives a complete description of the system. For $\mathcal{C}$ $=0$, the system does not have a complete set of eigenvectors and the growth is nonmodal. For intermediate values of $\mathcal{C}$ we have shown that relative importance of the modal and nonmodal behavior is time scale dependent. Our main conclusion is that for a given time scale, there is a $\mathcal{C}_{\text {crit }}$ such that for $\mathcal{C}<C_{\text {crit }}$ the behavior is nonmodal and for $\mathcal{C}>\mathcal{C}_{\text {crit }}$ the behavior is modal. Detailed results supporting this conclusion are the following.

(1) The pseudospectrum of the Hasegawa-Wakatani system shows that for small $\mathcal{C}$, nonmodal growth is larger than the modal growth predicted by the spectrum; this nonmodal 
(a)

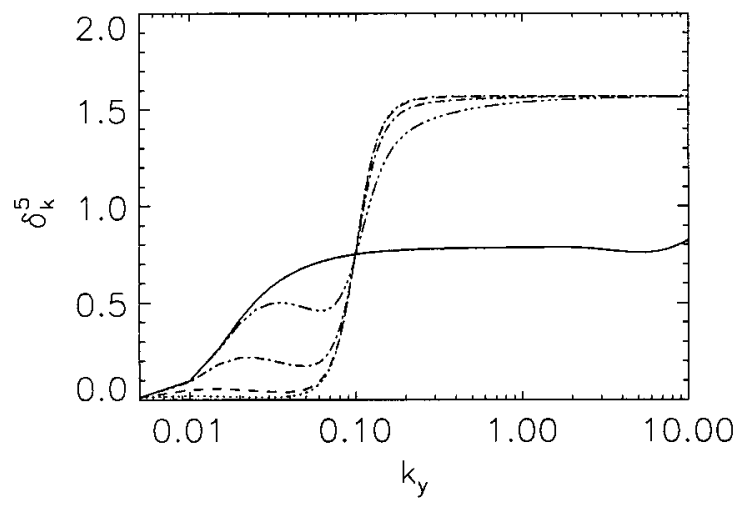

(c)

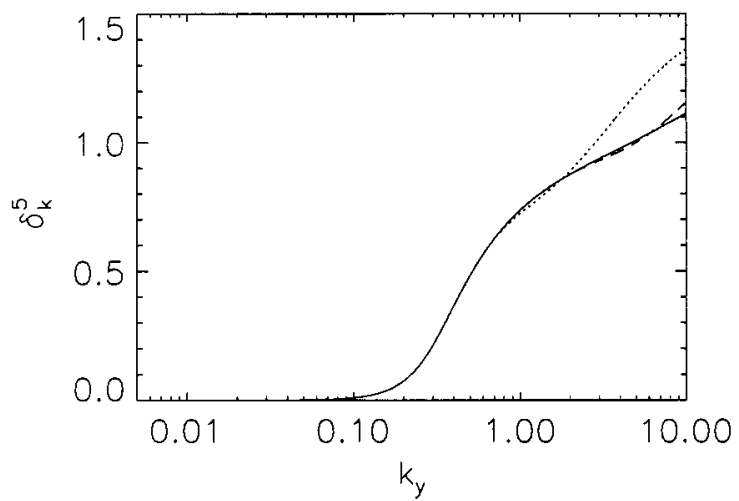

(b)

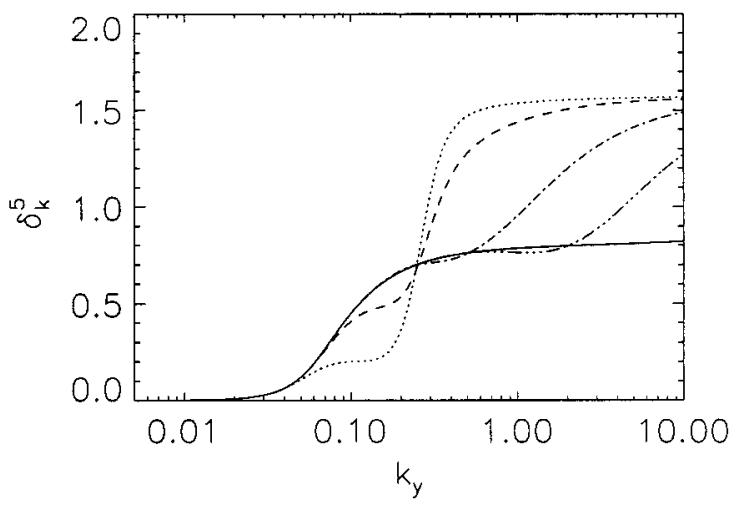

(d)

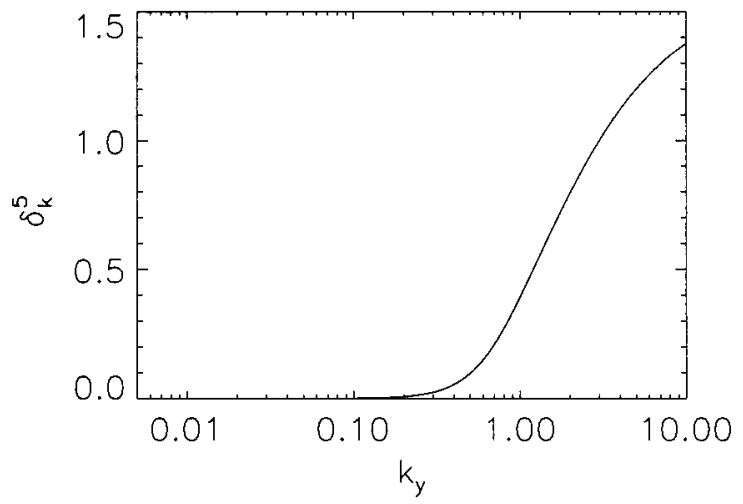

FIG. 8. Ensemble averaged linear phase shift $\delta_{\mathbf{k}}^{5}(t) \quad(t=10, t=20, t=50$, and $t=100)$ as a function of $k_{y}$ for (a) $\mathcal{C}=10^{-5},($ b) $\mathcal{C}$ $=10^{-3}$, (c) $\mathcal{C}=0.1$, and (d) $\mathcal{C}=1$. Here $\rho_{s}=0.005, N=2000$, and $k_{x} \equiv 0$.

growth occurs at different wave numbers than the maximum modal growth.

(2) For $\mathcal{C} \ll 1$, drift waves with $\beta_{0} \approx 0$ have nonmodal growth rates comparable to the modal growth rates of the most unstable drift waves $(C=0.1)$.

(3) The nonmodal behavior is generally a broad-spectrum phenomenon. A consequence of this point is that energy growth averaged over random initial conditions is greater for drift waves with $\mathcal{C} \ll 1$ than for drift waves with $\mathcal{C} \sim 1$ where narrow-spectrum modal behavior is dominant.

(3) Fluctuations that grow fastest initially maintain strong growth only in the case $\mathcal{C}=10^{-5}$. A consequence of this is that even for $\mathcal{C}=1$, where the behavior is mostly normal, the linear behavior on very short time scales $(t \ll 1)$ differs from the normal mode analysis.

These conclusions suggest that two properties of the nonlinear simulations of the Hasegawa-Wakatani, which were not explained by the normal mode analysis, should be examined again in the light of the nonmodal analysis. The first is the effect of the adiabatic parameter $\mathcal{C}$ on the time required for a saturated turbulent state to be reached. The saturation time was found to decrease for small $\mathcal{C}$ [20]. As the modal growth rate decreases for small $\mathcal{C}$, the decrease in saturation time was previously attributed to nonlinear effects. However, we have seen two mechanisms that might account for this behavior for small $\mathcal{C}$, (i) the nonmodal growth rates are not small and (ii) the nonmodal instability is broad spectrum.
These mechanisms could have been factors in the numerical stability considerations that limited the nonlinear simulations to values of $\mathcal{C}$ that were not too small [20]. The other property attributed to nonlinear effects was the saturation level, which was found to increase for small $\mathcal{C}$ and to be due primarily to density fluctuations [20]. This feature can be explained by the algebraic growth of density fluctuations that is present for small $\mathcal{C}$. Another possible consequence of nonmodal analysis is that the nonmodal finite-time phase shifts could help explain the enhancement of transport for certain wave numbers at given adiabaticity parameters [33].

\section{ACKNOWLEDGMENTS}

This work was supported by the CNPq (Conselho Nacional de Desenvolvimento Científico e Tecnológico) under Grant No. 381737/97-73, and by FAPESP (Fundação de Amparo à Pesquisa do Estado de São Paulo) under Grant No. 96/5388-0.

\section{APPENDIX}

We introduce new variables $\psi_{\mathbf{k}}$ defined by

$$
\psi_{\mathbf{k}}=\mathbf{M}_{\mathbf{k}}^{1 / 2} \mathbf{u}_{\mathbf{k}}
$$

where 


$$
\mathbf{M}_{\mathbf{k}}=\frac{1}{2}\left[\begin{array}{cc}
k^{2} & 0 \\
0 & 1
\end{array}\right]
$$

The advantage of these variables is that $\|\mathbf{u}\|=\|\psi\|_{2}$ where $\|\cdot\|$ is the energy norm and $\|\cdot\|_{2}$ is the usual root mean square norm, allowing the use of standard methods to compute the various energy growth ratios. Equation (7) becomes

$$
\frac{d}{d t} \psi_{\mathbf{k}}=\mathbf{B}_{\mathbf{k}} \psi_{\mathbf{k}},
$$

where

$$
\mathbf{B}_{\mathbf{k}}=\mathbf{M}^{1 / 2} \mathbf{A}_{\mathbf{k}} \mathbf{M}^{-1 / 2} .
$$

Note that since Eq. (A4) is a similarity transform $\mathbf{A}$ and $\mathbf{B}$ have the same eigenvalues and hence the same modal growth properties.

The energy growth ratios $\xi$ and growth rates $\beta$ are computed as follows. First,

$$
\beta_{0}=\max _{z \in \Lambda(\mathbf{B})} \operatorname{Re} z,
$$

and $\xi_{0}(t)=e^{\beta_{0} t}$. Then,

$$
\xi_{1}(t)=\left\|e^{\mathbf{B} t}\right\|_{2}=\max _{\mathbf{k}}\left\|e^{\mathbf{B}_{\mathbf{k}^{t}}}\right\|_{2} .
$$

Note that since there is no mode coupling only the matrix exponential of $2 \times 2$ matrices must be calculated. Then,

$$
\xi_{2}(t)=\left\|e^{\mathbf{B} t} y\right\|_{2},
$$

where $y$ is the left eigenvector of $\mathbf{B}$ whose eigenvalue has real part $\beta_{0}$ [3]. Then,

$$
\xi_{3}(t)=\left\|e^{\mathbf{B} t} v\right\|_{2},
$$

where $v$ is the leading right singular vector of $e^{\mathbf{B} \tau}$ ([26], Sec. 2.5.3). Then,

$$
\xi_{4}(t)=\left\|e^{\mathbf{B} t} w\right\|_{2},
$$

where $w$ is the leading eigenvector of $\left(\mathbf{B}^{\dagger}+\mathbf{B}\right)$ [2]

The growth rate ratio $\xi_{5}(t)$ is computed by considering an ensemble of initial conditions $\psi(0)$. In particular, suppose that the mean of the ensemble is zero,

$$
\langle\psi(0)\rangle=0,
$$

and that the covariance is given by

$$
\left\langle\psi(0) \psi(0)^{\dagger}\right\rangle=\mathbf{Q},
$$

where $\mathbf{Q}$ is the covariance matrix; \langle\rangle denotes ensemble average. Then, the ensemble averaged initial energy is

$$
\left\langle\|\psi(0)\|_{2}^{2}\right\rangle=\left\langle\operatorname{tr} \psi(0) \psi(0)^{\dagger}\right\rangle=\operatorname{tr} \mathbf{Q},
$$

and the expected energy at time $t$ is

$$
\begin{aligned}
\left\langle\|\psi(t)\|^{2}\right\rangle & =\left\langle\operatorname{tr} \psi(t) \psi(t)^{\dagger}\right\rangle=\left\langle\operatorname{tr} e^{\mathbf{B} t} \psi(0) \psi(0)^{\dagger} e^{\mathbf{B}^{\dagger} t}\right\rangle \\
& =\operatorname{tr} e^{\mathbf{B}^{\dagger} t} \mathbf{Q} e^{\mathbf{B} t} .
\end{aligned}
$$

The simplest choice of $\mathbf{Q}$ and the one we use here is $\mathbf{Q}$ $=\mathbf{I} / \mathrm{tr} \mathbf{I}$, i.e., uncorrelated random initial conditions with expected unit initial energy and therefore

$$
\xi_{5}^{2}(t)=\frac{1}{2 N^{2}} \sum_{\mathbf{k}} \operatorname{tr} e^{\mathbf{B}_{\mathbf{k}}^{\dagger} t} e^{\mathbf{B}_{\mathbf{k}} t} .
$$

Unlike the other energy growth ratios, $\xi_{5}(t)$ depends on all the wave numbers of the system. The quantity $\operatorname{tr} e^{\mathbf{B}^{\dagger} t} e^{\mathbf{B} t}$ converges as more modes are included $(N \rightarrow \infty)$ for any $t>0$. Therefore $\xi_{5}(t)$ depends on $N$ in two ways: (i) the truncation involved in the calculation of $\operatorname{tr} e^{\mathbf{B}^{\dagger} t} e^{\mathbf{B} t}$ and (ii) the normalizing factor $N^{-2} / 2$. While the number of modes used here, $N=32$, gives reasonable approximations to the quantity $\operatorname{tr} e^{\mathbf{B}^{\dagger} t} e^{\mathbf{B} t}$, we do not claim convergence in all cases. For instance, for $\mathcal{C}=1$ and $\mathcal{C}=10^{-5}$ the percentage error between $\operatorname{tr} e^{\mathbf{B}^{\dagger}} e^{\mathbf{B}} \quad(t=100)$ using $N=32$ and $N=64$ is $\left(3.9 \times 10^{-5}\right) \%$ and $7.8 \%$ respectively. Therefore the choice of $N=32$ in the calculation of $\xi_{5}$ is an assumption on the energy spectrum of the random initial conditions.
[1] P. G. Drazin and W. H. Reid, Hydrodynamic Stability (Cambridge University Press, Cambridge, England, 1981).

[2] L. N. Trefethen, A. E. Trefethen, and S. C. Reddy, Science 261, 578 (1993).

[3] B. F. Farrell and P. Ioannou, J. Atmos. Sci. 53, 2025 (1996).

[4] K. M. Butler and B. F. Farrell, Phys. Fluids A 4, 1637 (1992).

[5] B. F. Farrell, J. Atmos. Sci. 42, 163 (1988).

[6] R. Buizza and T. N. Palmer, J. Atmos. Sci. 52, 1434 (1995).

[7] T. DelSole and B. F. Farrell, J. Atmos. Sci. 53, 1781 (1996).

[8] S. C. Reddy, P. J. Schmid, and D. S. Henningson, SIAM (Soc. Ind. Appl. Math.) J. Appl. Math. 1, 15 (1993).

[9] J. S. Baggett, T. A. Driscoll, and L. N. Trefethen, Phys. Fluids 7, 833 (1995).

[10] B. F. Farrell and P. J. Ioannou, Phys. Fluids 8, 1257 (1996).

[11] R. J. Goldston and P. H. Rutherford, Introduction to Plasma Physics (Institute of Physics Publishing, Bristol, 1995).
[12] J. P. Freidberg, Ideal Magnetohydrodynamics (Plenum Press, New York, 1987).

[13] H. Tasso and S. J. Camargo, Nuovo Cimento B 107, 733 (1992).

[14] D. Borba et al., Phys. Plasmas 1, 3151 (1994).

[15] G. D. Chagelishvili, A. D. Rogava, and D. Tsiklauri, Phys. Plasmas 4, 1182 (1997).

[16] B. Scott, Plasma Phys. Controlled Fusion 39, 1635 (1997).

[17] A. J. Wootton et al., Phys. Fluids B 2, 2879 (1990).

[18] A. Hasegawa and M. Wakatani, Phys. Rev. Lett. 50, 682 (1985).

[19] M. Wakatani and A. Hasegawa, Phys. Fluids 27, 611 (1984).

[20] S. J. Camargo, D. Biskamp, and B. D. Scott, Phys. Plasmas 2, 48 (1995)

[21] D. Biskamp, S. J. Camargo, and B. D. Scott, Phys. Lett. A 186, 239 (1994). 
[22] A. E. Koniges, J. A. Crotinger, and P. H. Diamond, Phys. Fluids B 4, 2685 (1992).

[23] D. Biskamp and A. Zeiler, Phys. Rev. Lett. 74, 706 (1995).

[24] A. Zeiler, D. Biskamp, and J. F. Drake, Phys. Plasmas 3, 3947 (1996).

[25] A. Hasegawa and K. Mima, Phys. Fluids 21, 87 (1978).

[26] G. H. Golub and C. F. Van Loan, Matrix Computations, 3rd ed. (The Johns Hopkins University Press, Baltimore, 1996).

[27] P. R. Halmos, A Hilbert Space Problem Book (Van NostrandReinhold, New York, 1967).
[28] L. N. Trefethen, SIAM (Soc. Ind. Appl. Math.) Rev. 39, 383 (1997).

[29] J. H. Wilkinson, The Algebraic Eigenvalue Problem (Clarendon Press, Oxford, England, 1965).

[30] B. F. Farrell, J. Atmos. Sci. 46, 1193 (1989).

[31] Z. Toth and E. Kalnay, Bull. Am. Meteorol. Soc. 74, 2317 (1993).

[32] F. Molteni, R. Buizza, T. N. Palmer, and T. Petroliagis, Q. J. R. Meteorol. Soc. 122, 73 (1996).

[33] C. P. Ritz, E. J. Powers, and R. D. Bengtson, Phys. Fluids B 1, 153 (1989). 